\title{
Alantolactone induces apoptosis of human cervical cancer cells via reactive oxygen species generation, glutathione depletion and inhibition of the Bcl-2/Bax signaling pathway
}

\author{
YAN JIANG $^{1}$, HANJIE XU $^{2}$ and JIAFEI WANG ${ }^{1}$ \\ Departments of ${ }^{1}$ Gynecological Oncology and ${ }^{2}$ Gynecology and Obstetrics, Anhui Cancer Hospital, \\ Hefei, Anhui 230031, P.R. China
}

Received March 18, 2015; Accepted December 17, 2015

DOI: $10.3892 / \mathrm{ol} .2016 .4511$

\begin{abstract}
Alantolactone is the active ingredient in frankincense, and is extracted from the dry root of elecampane. It has a wide variety of uses, including as an insect repellent, antibacterial, antidiuretic, analgesic and anticancer agent. In addition, alantolactone induces apoptosis of human cervical cancer cells, however, its mechanism of action remains to be elucidated. Therefore, the present study investigated whether alantolactone was able to induce apoptosis of human cervical cancer cells, and its potential mechanisms of action were analyzed. Treatment of HeLa cells with alantolactone $(0,10$, $20,30,40,50$ and $60 \mu \mathrm{M})$ for $12 \mathrm{~h}$ significantly inhibited growth in a dose-dependent manner. Cells treated with $30 \mu \mathrm{M}$ of alantolactone for $0,3,6$ and $12 \mathrm{~h}$ demonstrated marked induction of apoptosis in a time-dependent manner. Treatment of HeLa cells with $30 \mu \mathrm{M}$ of alantolactone for $0,3,6$ and $12 \mathrm{~h}$ significantly induced the generation of reactive oxygen species (ROS) and inhibited glutathione (GSH) production in HeLa cells in a dose-dependent manner. Alantolactone additionally markedly inhibited the Bcl-2/Bax signaling pathway in HeLa cells. Therefore, administration of alantolactone induced apoptosis of human cervical cancer cells via ROS generation, GSH depletion and inhibition of the Bcl-2/Bax signaling pathway.
\end{abstract}

\section{Introduction}

Cervical carcinoma is a commonly observed malignant tumor of the female reproductive system, which occurs in the vagina and cervical canals. It is the second most common gynecological tumor globally, following breast cancer (1).

Correspondence to: Dr Yan Jiang, Department of Gynecological Oncology, Anhui Cancer Hospital, 107 East Road, Hefei, Anhui 230031, P.R. China

E-mail: yanjiangah@163.com

Key words: alantolactone, cervical cancer, reactive oxygen species, glutathione, B-cell lymphoma 2/Bcl-2-associated X protein
In the developing world, cervical carcinoma belongs to the common multiple gynecological tumor (2). Approximately 500,000 new cervical cancer cases are diagnosed annually, accounting for $5 \%$ of all diagnosed cases of cancer worldwide, and $80 \%$ of these diagnoses occur in developing countries (3).

The cause of cervical carcinoma remains to be elucidated. A large body of epidemiological data and associated studies has concluded that a major risk factor is infection with the sexually transmitted human papilloma virus (HPV), particularly HPV16 and HPV18 (4). However, not all HPV infections progress to cause cervical carcinoma (5). Alternative risk factors should also be considered, including sexual disorders, smoking and malnutrition, which may partly explain the high incidence of cervical carcinoma in the lower economic levels of society (6). It has been reported that cervical carcinoma may be associated with dietary factors, and certain studies have suggested that micronutrients, including folic acid, vitamin $A$, vitamin $C, \beta$-carotene and vitamin $E$, may have a role in the prevention of human malignant tumors, including cervical carcinoma (7). These above-mentioned elements are antioxidants (8). During the process of cervical carcinoma occurrence, reactive oxygen species (ROS) may cause dysregulation of the chemical properties of the body, leading to the damaging of DNA and proteins, eventually causing cancer. In contrast to ROS, the above-mentioned micronutrients, which demonstrate intracellular antioxidant ability, cause active oxygen inactivation, preventing oxidative damage and thus reducing the occurrence of cervical carcinoma (9).

Inula helenium L. belongs to the Compositae family and the genus Inula. It is a perennial herbaceous plant that is widely distributed across Xinjiang and other regions in China, and in certain areas may be cultivated (10). The dry root of Inula helenium L. may have medicinal properties, including as an expectorant, insect repellent and antidiuretic agent (11). Alantolactone is isolated from the elecampane root, and is one of the main terpene lactone compounds. It has been confirmed to exert antitumor, anti-inflammatory and antibacterial effects (12-14). Previous findings have shown that alantolactone was able to induce U87 glioma cell apoptosis by mitochondrial injury, via an increase in ROS production (15). 
Alantolactone has been previously identified to be able to induce apoptosis of liver cancer cells . The primary mechanism that allows achievement of this is via adjustment of Bcl-2 protein expression and activation of the caspase family. However, the underlying molecular mechanism through which alantolactone is able to induce anticancer effects and apoptosis in human cervical cancer cells remains to be elucidated. The present study aimed to identify the underlying molecular mechanism and signaling pathway that leads to anticancer effects and apoptosis in human cervical cancer cells. The present study identified ROS and glutathione (GSH) as key effectors, which may lead to activation of the $\mathrm{Bcl}-2 / \mathrm{Bax}$ signaling pathway.

\section{Materials and methods}

Reagents. Dulbecco's modified Eagle's medium (DMEM), fetal bovine serum (FBS) and 3-(4,5-dimethylthiazol2-yl)-2,5-diphenyltetrazolium bromide (MTT) were obtained from Sigma-Aldrich (St. Louis, MO, USA). The apoptosis assay kit, ROS-sensitive dichlorodihydrofluorescin diacetate (H2DCFDA) kit, GSH and oxidized GSH (GSSG) kits were purchased from Nanjing KeyGen Biotech Co., Ltd. (Nanjing, China). BCA protein assay was obtained from Beyotime Institute of Biotechnology (Haimen, China).

Cell culture. The HeLa human cervical cancer cell line was obtained from the Cell Bank of the Chinese Academy of Sciences (Shanghai, China). HeLa cells were cultured in DMEM supplemented with $10 \%$ FBS, $0.03 \%$ L-glutamine (Thermo Fisher Scientific, Inc., Waltham, MA, USA), penicillin (100 U/ml; Sangon Biotech Co., Ltd., Shanghai, China) and streptomycin (100 $\mu \mathrm{g} / \mathrm{ml}$; Sangon Biotech Co., Ltd.), and maintained at $37^{\circ} \mathrm{C}$ with $5 \% \mathrm{CO}_{2}$ in a humidified atmosphere.

MTT assay. Cell growth inhibition was measured using MTT assay as described previously (16). Briefly, HeLa cells were dispensed into 96-well flat bottom microtiter plates (Thermo Fisher Scientific, Inc.) and treated with alantolactone (Sigma-Alrich; 0, 10, 20, 30, 40, 50 and $60 \mu \mathrm{M}$ ) for $12 \mathrm{~h}$. Subsequently, MTT solution was added to each well for $4 \mathrm{~h}$. Following incubation with MTT, $150 \mu \mathrm{l}$ dimethylsulfoxide (GE Healthcare, Logan, UT, USA) was added to dissolve the formazan crystals. Optical density was measured with a microplate reader at $570 \mathrm{~nm}$ (Multiskan MK3; Thermo Fisher Scientific, Inc.). Cell viability was calculated based on the

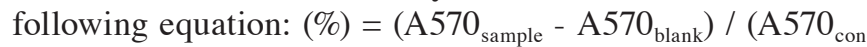
trol $\left.-\mathrm{A} 570_{\text {blank }}\right) \times 100$.

Observation of apoptosis. Cell apoptosis was measured by flow cytometry. Briefly, HeLa cells were dispensed into 6-well plates (Thermo Fisher Scientific, Inc.) at a density of $1 \times 10^{6}$ per flask. Following $24 \mathrm{~h}$ of incubation, HeLa cells were treated with $30 \mu \mathrm{M}$ of alantolactone for $0,3,6$ and $12 \mathrm{~h}$. The cells were harvested using trypsin (Wuhan Amyjet Scientific Co., Ltd., Wuhan, China) and washed with phosphate-buffered saline (Sangon Biotech Co., Ltd.). HeLa cells were resuspended in $500 \mu 1$ of binding buffer (Sangon Biotech Co., Ltd.), and subsequently cultured with $5 \mu \mathrm{l}$ Annexin $\mathrm{V}$-fluorescein isothiocyanate and propidium iodide in the dark for $15 \mathrm{~min}$, according to the manufacturer's protocol (Nanjing KeyGen Biotech Co., Ltd.). The samples were analyzed using FACScan ${ }^{\mathrm{TM}}$ and Epics XL flow cytometers (Beckman Coulter, Inc., Brea, CA, USA).

Measurement of ROS. Levels of ROS were measured using the H2DCFDA kit. Briefly, HeLa cells were dispensed into 96-well flat bottom microtiter plates and treated with $30 \mu \mathrm{M}$ of alantolactone for $0,3,6$ and $12 \mathrm{~h}$. HeLa cells were incubated with $5 \mu \mathrm{M}$ H2DCFDA for $0.5-1 \mathrm{~h}$. The fluorescence intensity of ROS was measured using a microplate reader (Multiskan MK3) at 485/535 $\mathrm{nm}$.

Measurement of GSH and GSSG. Levels of GSH and GSSG were determined with GSH and GSSG assay kits (Beyotime Institute of Biotechnology). Briefly, HeLa cells were dispensed into 96-well flat bottom microtiter plates and treated with $30 \mu \mathrm{M}$ of alantolactone for $0,3,6$ and $12 \mathrm{~h}$. Following treatment, the levels of GSH and GSSG were measured according to the manufacturer's protocol of the GSH and GSSG kits.

Western blot analysis. HeLa cells were dispensed into 6-well flat bottom microtiter plates and treated with $30 \mu \mathrm{M}$ of alantolactone for $0,3,6$ and $12 \mathrm{~h}$. Following plasma treatment, HeLa cells were lysed in radioimmunoprecipitation assay buffer $[0.1 \%$ sodium dodecyl sulfate (SDS); $150 \mathrm{mM}$ $\mathrm{NaCl} ; 1 \% \mathrm{NP}-40 ; 25$ mM Tris-HCl (pH 7.6); 1\% sodium deoxycholate]. Subsequently, lysed solution was spun in a centrifuge at $12,000 \mathrm{x}$ for $10 \mathrm{~min}$ at $4^{\circ} \mathrm{C}$. Equal amounts of protein were obtained using the BCA protein assay. Protein $(40 \mu \mathrm{g})$ was separated using $12 \%$ SDS polyacrylamide gel electrophoresis ( $75 \mathrm{~V}$ for $45 \mathrm{~min}$ and $110 \mathrm{~V}$ for $75 \mathrm{~min}$ ) and blotted onto nitrocellulose membranes (EMD Millipore, Billerica, MA, USA). Cell lysates were immunoblotted using mouse anti-human anti-Bcl-2 (catalog no., sc-7382) and mouse anti-human anti-Bax (sc-20067; both diluted 1:1,000) monoclonal antibodies (Santa Cruz Biotechnology, Inc., Dallas, TX, USA). The proteins were visualized using a goat anti-rabbit immunoglobulin G PerCP-Cy5.5 conjugated secondary antibody (dilution, 1:3,000; catalog no., sc-45101; Santa Cruz Biotechnology, Inc.) and Amersham ECL Western Blotting Detection Reagent (GE Healthcare Life Sciences, Chalfont, UK).

Statistical analysis. The results are shown as the mean \pm standard deviation, confirmed by at least three independent experiments. Statistical comparisons were performed using one-way analysis of variance. Statistical analyses were performed using SPSS version 13.0 (SPSS, Inc., Chicago, IL, USA). $\mathrm{P}<0.05$ was considered to indicate a statistically significant difference.

\section{Results}

Alantolactone inhibits the growth of HeLa cells. The chemical structure of alantolactone (purity of $98 \%$ calculated by high-performance liquid chromatography) is shown in Fig. 1. The anticancer effects of alantolactone on the growth of HeLa cells were measured using an MTT assay. Administration of alantolactone $(0,10,20,30,40,50$ and $60 \mu \mathrm{M})$ for 


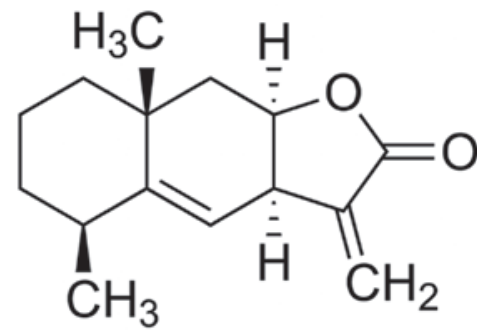

Figure 1. Chemical structure of alantolactone.

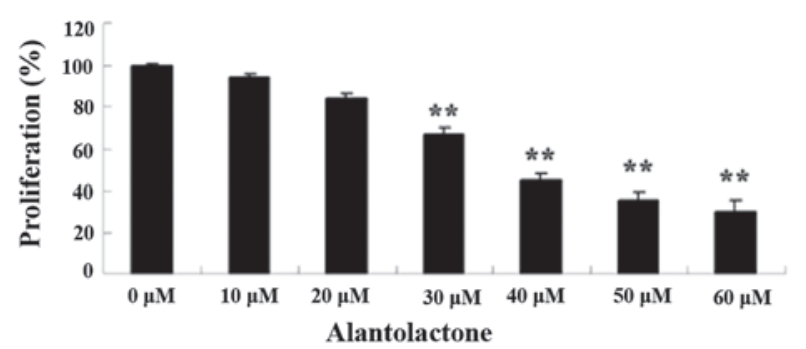

Figure 2. Alantolactone inhibits growth of HeLa cells. ${ }^{* *} \mathrm{P}<0.01$ compared with $0 \mu \mathrm{M}$ treatment group. The results are presented as the mean \pm standard deviation of at least three independent experiments.

$12 \mathrm{~h}$ inhibited the growth of HeLa cells in a dose-dependent manner (Fig. 2). Therefore, $30 \mu \mathrm{M}$ of alantolactone was used in the subsequent experiments.

Alantolactone induces the apoptosis of HeLa cells. To examine the anticancer effects of alantolactone on apoptosis, HeLa cells were treated with $30 \mu \mathrm{M}$ of alantolactone for $0,3,6$ and $12 \mathrm{~h}$, and apoptosis was measured with flow cytometry. As shown in Fig. 3, treatment with alantolactone induced apoptosis of HeLa cells in a time-dependent manner. The results of the present study suggested that alantolactone-induced cell apoptosis indicated early apoptosis prior to the $6 \mathrm{~h}$ time point.

Alantolactone induces ROS generation in HeLa cells. It was verified that the levels of ROS generation have a significant role in the anticancer effects of alantolactone on HeLa cells. Levels of ROS were measured using the H2DCFDA kit. Pretreatment of HeLa cells with $30 \mu \mathrm{M}$ of alantolactone for $0,3,6$ and $12 \mathrm{~h}$ induced ROS generation in HeLa cells in a time-dependent manner (Fig. 4). The results of the present study showed that alantolactone effectively induced ROS generation prior to the $6 \mathrm{~h}$ time point.

Alantolactone inhibits GSH and GSSG production in HeL a cells. To investigate whether the levels of GSH and GSSG generation had significant roles in the anticancer effects of alantolactone on HeLa cells, the levels of GSH and GSSG were measured using commercial kits. The results of the present study showed that alantolactone inhibited GSH generation in a time-dependent manner (Fig. 5). Following treatment with alantolactone for $6 \mathrm{~h}, \mathrm{GSH}$ generation was effectively inhibited. However, the levels of GSSG generation did not demonstrate a statistically significant difference in any of the experimental groups.

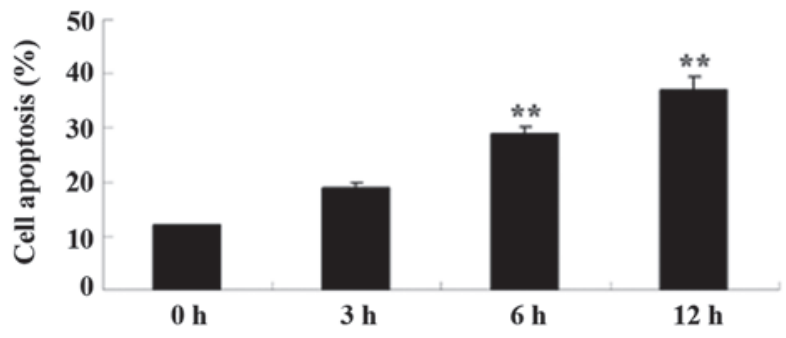

Figure 3. Alantolactone induces apoptosis of HeLa cells. ${ }^{* *} \mathrm{P}<0.01$ compared with $0 \mu \mathrm{M}$ treatment group. The results are presented as the mean \pm standard deviation of at least three independent experiments.

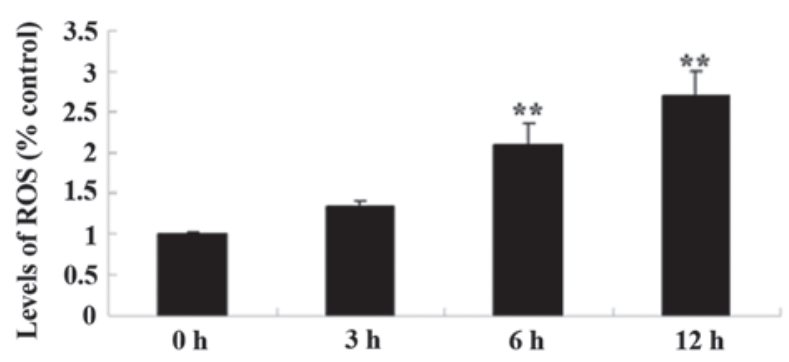

Figure 4. Alantolactone induces ROS generation in HeLa cells. ${ }^{* *} \mathrm{P}<0.01$ compared with $0 \mu \mathrm{M}$ treatment group. The results are presented as the mean \pm standard deviation of at least three independent experiments. ROS, reactive oxygen species.
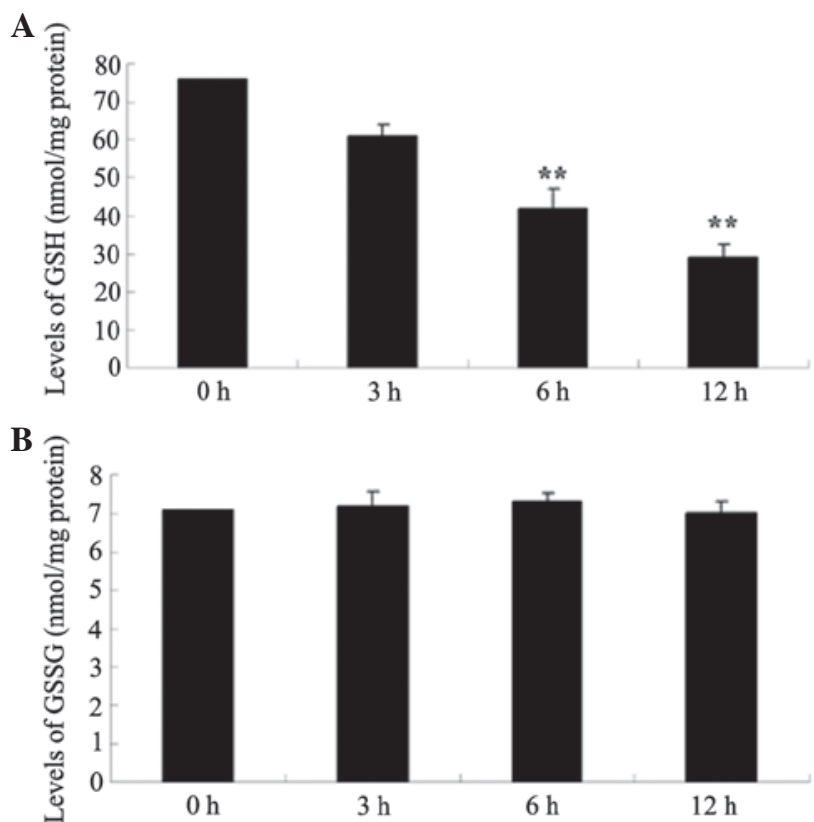

Figure 5. Alantolactone inhibits GSH and GSSG production in HeLa cells Alantolactone inhibits (A) GSH and (B) GSSG production in HeLa cells. ${ }^{* *} \mathrm{P}<0.01$ compared with $0 \mu \mathrm{M}$ treatment group. The results are presented as the mean \pm standard deviation of at least three independent experiments. GSH, glutathione; GSSG, oxidized glutathione.

Alantolactone inhibits the Bcl-2/Bax signaling pathway in HeLa cells. To investigate the Bcl-2/Bax signaling pathway in alantolactone-induced apoptosis, Bcl-2 and Bax protein expression was determined using western blot analysis. As shown in Fig. 6, Bcl-2 and Bax protein expression were inhibited and increased, respectively, in a time-dependent manner following 
A
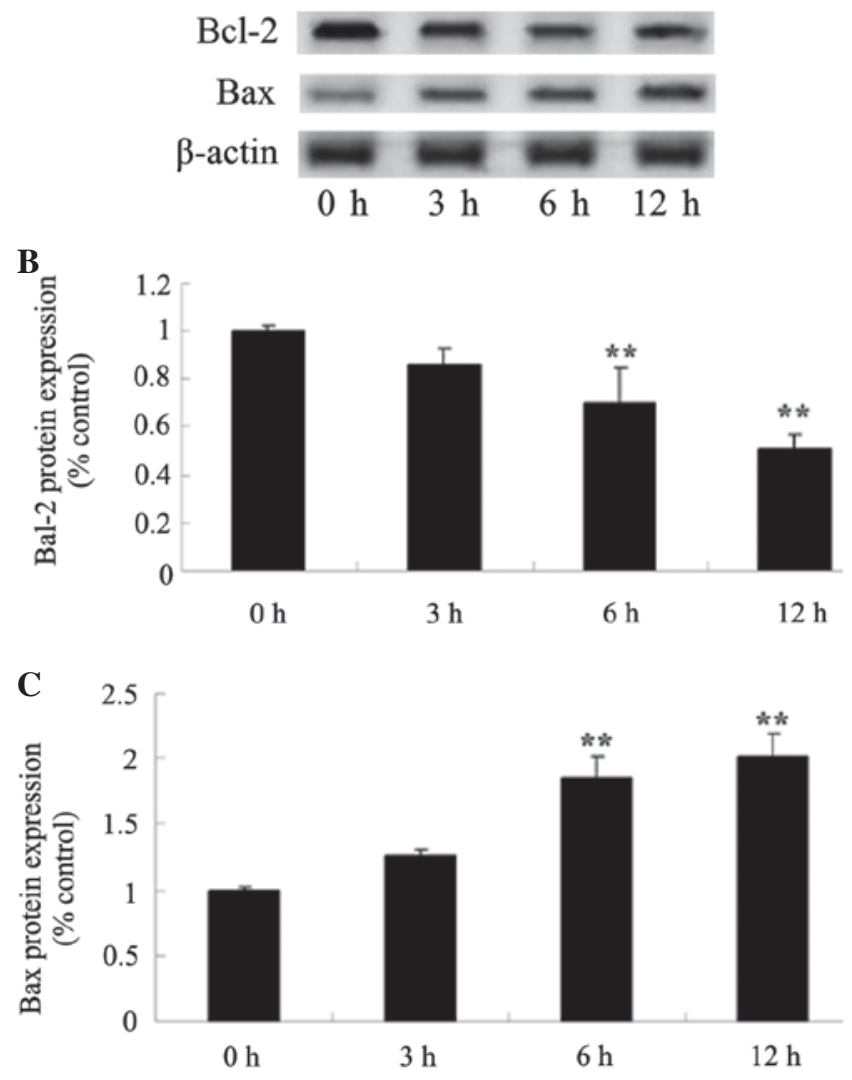

Figure 6. Alantolactone inhibits the Bcl-2/Bax signaling pathway in HeLa cells. Alantolactone inhibits Bcl-2 and Bax protein expression, demonstrated by (A) western blot analysis and (B and C) statistical analysis of Bcl-2 and Bax protein expression in HeLa cells. ${ }^{* *} \mathrm{P}<0.01$ compared with $0 \mu \mathrm{M}$ treatment group. The results are presented as the mean \pm standard deviation of at least three independent experiments. Bcl-2, B-cell lymphoma 2; Bax, Bcl-2-associated X protein.

treatment with $30 \mu \mathrm{M}$ of alantolactone for $0,3,6$ and $12 \mathrm{~h}$. Following treatment with alantolactone for $6 \mathrm{~h}$, the Bcl-2/Bax signaling pathway was effectively inhibited.

\section{Discussion}

Cervical carcinoma is one of the primary diseases that present a serious threat to women's health. In terms of global malignant tumor occurrence, the incidence of cervical carcinoma is second only to breast cancer. Cervical carcinoma is one of the leading causes of cancer in developing countries, whereas in developed countries, it is ranked below breast cancer and third overall (1). According to the World Health Organization, there are 500,000 new cases of cervical carcinoma worldwide each year, and $80 \%$ of these cases occur in developing countries (17). There are a total of 140,000 new cases of cervical carcinoma each year in China (18). The results of the present study indicated that administration of alantolactone was able to inhibit growth and induce the apoptosis of HeLa cells in a dose or time-dependent manner. In addition, Jarrett et al and Shi et al demonstrated clearly that alantolactone inhibited the proliferation of HCT- 8 human colon adenocarcinoma cells $(16,19)$. Khan et al $(20)$ reported that alantolactone induced apoptosis in HepG2 hepatoma cells.
Chronic inflammation has long been considered to be a risk factor for a variety of human malignant tumors, particularly cervical carcinoma. The chronic inflammation hypothesis emphasizes that the ROS produced by phagocytes leads to cytotoxicity and mutation (21). In the event that a chronic inflammatory tissue contains large amounts of nitric oxide (NO) and derivative ROS, NO and ROS may cause direct and indirect damage to DNA and other genetic material (22). The balance and interaction between NO and ROS has a significant role in the etiology of a tumor (23). The results of the present study regarding the underlying mechanism of alantolactone action on HeLa cells have implications for the promotion of ROS-mediated curative effects. Khan et al (15) demonstrated that alantolactone induced cell apoptosis via GSH depletion and ROS generation in glioblastoma cells. Zhang et al (24) reported that alantolactone induced cell apoptosis via ROS generation in RKO cells.

The onset of cervical carcinoma may be associated with long-term estrogen stimulation, as cervical carcinoma is a type of hormone dependent tumor (25). It has been proposed that androgen transfer into estrogen in fat tissue is involved in an increase in the levels of estrogen synthesis (26). Previous studies identified that Japanese fat consumption and cervical carcinoma incidence was lower compared with Finland. Finnish hydrogen peroxide levels in normal endometrium and GSH-peroxidase (Px) activity were lower compared with that of Japanese individuals. However, in Japan and Finland, patients exhibiting cervical carcinoma had increased endometrial lipid peroxide (LPO) levels compared with a healthy group, while superoxide dismutase and GSH-Px activity was reduced compared with a healthy group. This result demonstrated that cervical carcinoma and multiple quantity increased the intake of fat, resulting in the lipid peroxidation and antioxidant system being weakened $(27,28)$. In addition, a previous study demonstrated a significant increase in GSH-Px activity in cervical carcinoma tissue, and in well-differentiated adenocarcinomas this association was more marked compared with moderate or poorly differentiated adenocarcinomas (29). In the present study, the levels of GSH generation had significant roles in the anticancer effects of alantolactone on HeLa cells. Khan et al $(15,20)$ demonstrated that alantolactone was able to induce apoptosis of HepG2 cells and glioblastoma cells via GSH depletion.

The present study demonstrated that of the various genes involved in the regulation of cell apoptosis, Bcl-2 is particularly important, as it is regarded as one of the final common pathways of apoptosis regulation (30). Bcl-2 exhibits increased expression in a number of tumors, and is able to inhibit the natural apoptosis of cells, as well as suppress the apoptosis induced by many antitumor drugs, reducing their cytotoxicity. By contrast, Bax promotes apoptosis (31). The underlying mechanism of Bax activity does not block apoptosis directly, but inhibits the anti-apoptotic role of Bcl-2 (32). The results of the present study demonstrate that the anticancer effects of alantolactone on HeLa cells were associated with the Bcl-2/Bax signaling pathway. Administration of alantolactone may be capable of inhibiting the Bcl-2/Bax signaling pathway in HeLa cells. Recent studies have suggested that alantolactone likely increases the ratio of $\mathrm{Bcl}-2$ to Bax, therefore inducing apoptosis in cancer cells $(33,34)$.

In conclusion, the results of the present study provide evidence that alantolactone is capable of inducing apoptosis in 
human cervical cancer cells. Consequently, promotion of ROS generation, and inhibition of GSH generation and the Bcl-2/Bax signaling pathway induced the anticancer effects of alantolactone on apoptosis in HeLa cells. The results suggest that alantolactone is a promising Traditional Chinese Medicine that may be utilized for the treatment of cervical cancer. Additional investigation is required to verify the contribution of alantolactone to anticancer therapy in vitro and in vivo.

\section{References}

1. Yau Hsiung W and Abdul Kadir H: Leea indica ethyl acetate fraction induces growth-inhibitory effect in various cancer cell lines and apoptosis in ca ski human cervical epidermoid carcinoma cells. Evid Based Complement Alternat Med 2011: 293060, 2011.

2. Einck JP, Hudson A, Shulman AC, Yashar CM, Dieng MM, Diagne M, Gueye L, Gningue F, Gaye PM, Fisher BJ, et al: Implementation of a high-dose-rate brachytherapy program for carcinoma of the cervix in Senegal: A pragmatic model for the developing world. Int J Radiat Oncol Biol Phys 89: 462-467, 2014

3. Misra JS, Srivastava AN and Das V: Single life time cytological screening in high risk women as an economical and feasible approach to control cervical cancer in developing countries like India. Asian Pac J Cancer Prev 16: 859-862, 2015.

4. Di Gao Z, Pan Q, Lv H, Sun Y, Ma X, Qin Z and Sun YP: HPV genotypes in paraffin sections of non-cervical squamous cell carcinoma in Qingdao of China. Oncol Lett 5: 1219-1222, 2013.

5. Bigoni J, Gundar M, Tebeu PM, Bongoe A, Schäfer S, Fokom-Domgue J, Catarino R, Tincho EF, Bougel S, Vassilakos P and Petignat P: Cervical cancer screening in sub-Saharan Africa: A randomized trial of VIA versus cytology for triage of HPV-positive women. Int J Cancer 137: 127-134, 2015.

6. Gerli S, Bavetta F and Di Renzo GC: Antisepsis regimen in the surgical treatment of HPV generated cervical lesions: Polyhexamethylene biguanide vs chlorhexidine. A randomized, double blind study. Eur Rev Med Pharmacol Sci 16: 1994-1998, 2012.

7. Rodriguez EF, Reynolds JP, Jenkins SM, Winter SM, Henry MR and Nassar A: Atypical squamous cells of undetermined significance in patients with HPV positive DNA testing and correlation with disease progression by age group: An institutional experience. Int J Clin Exp Pathol 5: 428-435, 2012.

8. Xiang T, Du L, Pham P, Zhu B and Jiang S: Nelfinavir, an HIV protease inhibitor, induces apoptosis and cell cycle arrest in human cervical cancer cells via the ROS-dependent mitochondrial pathway. Cancer Lett 364: 79-88, 2015.

9. Singh M, Bhui K, Singh R and Shukla Y: Tea polyphenols enhance cisplatin chemosensitivity in cervical cancer cells via induction of apoptosis. Life Sci 93: 7-16, 2013.

10. Dupuis G, Mitchell JC and Towers GH: Reaction of alantolactone, an allergenic sesquiterpene lactone, with some amino acids. Resultant loss of immunologic reactivity. Can J Biochem 52: 575-581, 1974

11. Yao Y, Xia D, Bian Y, Sun Y, Zhu F, Pan B, Niu M, Zhao K, $\mathrm{Wu}$ Q, Qiao J, et al: Alantolactone induces G1 phase arrest and apoptosis of multiple myeloma cells and overcomes bortezomib resistance. Apoptosis 20: 1122-1133, 2015.

12. Zhang J, Li Y, Duan D, Yao J, Gao K and Fang J: Inhibition of thioredoxin reductase by alantolactone prompts oxidative stress-mediated apoptosis of HeLa cells. Biochem Pharmacol 2015 (In press)

13. Lim HS, Jin SE, Kim OS, Shin HK and Jeong SJ: Alantolactone from Saussurea lappa exerts antiinflammatory effects by inhibiting chemokine production and STAT1 phosphorylation in TNF- $\alpha$ and IFN- $\gamma$-induced in HaCaT cells. Phytother Res 29: 1088-1096, 2015

14. Ketai W, Huitao L, Yunkun Z, Xingguo C, Zhide H, Yucheng S and Xiao M: Separation and determination of alantolactone and isoalantolactone in traditional Chinese herbs by capillary electrophoresis. Talanta 52: 1001-1005, 2000

15. Khan M, Yi F, Rasul A, Li T, Wang N, Gao H, Gao R and Ma T: Alantolactone induces apoptosis in glioblastoma cells via GSH depletion, ROS generation, and mitochondrial dysfunction. IUBMB Life 64: 783-794, 2012.
16. Jarrett SG, Albon J and Boulton M: The contribution of DNA repair and antioxidants in determining cell type-specific resistance to oxidative stress. Free Radic Res 40: 1155-1165, 2006.

17. Liao S, Xiao S, Zhu G, Zheng D, He J, Pei Z, Li G and Zhou Y: CD38 is highly expressed and affects the PI3K/Akt signaling pathway in cervical cancer. Oncol Rep 32: 2703-2709, 2014.

18. Levin CE, Sharma M, Olson Z, Verguet S, Shi JF, Wang SM, Qiao YL, Jamison DT and Kim JJ: An extended cost-effectiveness analysis of publicly financed HPV vaccination to prevent cervical cancer in China. Vaccine 33: 2830-2841, 2015.

19. Shi Y, Bao YL, Wu Y, Yu CL, Huang YX, Sun Y, Zheng LH and Li YX: Alantolactone inhibits cell proliferation by interrupting the interaction between Cripto-1 and activin receptor type II A in activin signaling pathway. J Biomol Screen 16: 525-535, 2011.

20. Khan M, Li T, Ahmad Khan MK, Rasul A, Nawaz F, Sun $\mathrm{M}$, Zheng Y and Ma T: Alantolactone induces apoptosis in HepG2 cells through GSH depletion, inhibition of STAT3 activation, and mitochondrial dysfunction. BioMed Res Int 2013: 719858,2013

21. Yang J, Xiao YL, He XR, Qiu GF and Hu XM: Aesculetin-induced apoptosis through a ROS-mediated mitochondrial dysfunction pathway in human cervical cancer cells. J Asian Nat Prod Res 12: 185-193, 2010.

22. He L, Nan MH, Oh HC, Kim YH, Jang JH, Erikson RL, Ahn JS and Kim BY: Asperlin induces $\mathrm{G}_{2} / \mathrm{M}$ arrest through ROS generation and ATM pathway in human cervical carcinoma cells. Biochem Biophys Res Commun 409: 489-493, 2011.

23. Agostinelli E and Seiler N: Non-irradiation-derived reactive oxygen species (ROS) and cancer: Therapeutic implications. Amino Acids 31: 341-355, 2006.

24. Zhang Y, Bao YL, Wu Y, Yu CL, Huang YX, Sun Y, Zheng LH and Li YX: Alantolactone induces apoptosis in RKO cells through the generation of reactive oxygen species and the mitochondrial pathway. Mol Med Rep 8: 967-972, 2013.

25. Kim YT, Kim SW, Yoon BS, Cho HJ, Nahm EJ, Kim SH, Kim JH and Kim JW: Effect of intravenously administered iron sucrose on the prevention of anemia in the cervical cancer patients treated with concurrent chemoradiotherapy. Gynecol Oncol 105: 199-204, 2007.

26. Srivastava K, Paul S, Chufal KS, Shamsundar SD, Lal P, Pant MC, Bhatt M, Singh S and Gupta R: Concurrent chemoradiation versus radiotherapy alone in cervical carcinoma: A randomized phase III trial. Asia Pac J Clin Oncol 9: 349-356, 2013.

27. Fan S, Yu Y, Qi M, Sun Z, Li L, Yao G, Tashiro S, Onodera S and Ikejima T: P53-mediated GSH depletion enhanced the cytotoxicity of NO in silibinin-treated human cervical carcinoma HeLa cells. Free Radic Res 46: 1082-1092, 2012.

28. Kwaśniewska A, Tukendorf A and Semczuk M: Frequency of HPV infection and GSH levels in plasma of women with cervical dysplasia. Eur J Gynaecol Oncol 18: 196-199, 1997.

29. Moon HJ and Park WH: Butylated hydroxyanisole inhibits the growth of HeLa cervical cancer cells via caspase-dependent apoptosis and GSH depletion. Mol Cell Biochem 349: 179-186, 2011.

30. Chan HW, Liu T, Verdile G, Bishop G, Haasl RJ, Smith MA, Perry G, Martins RN and Atwood CS: Copper induces apoptosis of neuroblastoma cells via post-translational regulation of the expression of Bcl-2-family proteins and the tx mouse is a better model of hepatic than brain $\mathrm{Cu}$ toxicity. Int J Clin Exp Med 1: 76-88, 2008.

31. Li J, Wang H, Ma Z, Fan W, Li Y, Han B, Zhang Z and Wang J: TAT-Apoptin induces apoptosis in the human bladder cancer EJ cell line and regulates Bax, Bcl-2, caspase-3 and survivin expression. Exp Ther Med 3: 1033-1038, 2012.

32. Ji M, Yuan L, Lv X, Dong W and Peng X: EBP50 regulates the apoptosis of pancreatic cancer cells by decreasing the expression levels of Bcl-2. Exp Ther Med 8: 919-924, 2014.

33. Mi XG, Song ZB, Wu P, Zhang YW, Sun LG, Bao YL, Zhang Y, Zheng LH, Sun Y, Yu CL, et al: Alantolactone induces cell apoptosis partially through down-regulation of testes-specific protease 50 expression. Toxicol Lett 224: 349-355, 2014.

34. Zhao P, Pan Z, Luo Y, Zhang L, Li X, Zhang G, Zhang Y, Cui R, Sun $\mathrm{M}$ and Zhang $\mathrm{X}$ : Alantolactone induces apoptosis and cell cycle arrest on lung squamous cancer SK-MES-1 cells. J Biochem Mol Toxicol 29: 199-206, 2015. 